\title{
Bounds for Characteristic Roots of Matrices II
}

\section{Olga Taussky}

\begin{abstract}
This is a continuation of an earlier note (Duke Mathematical Journal, volume 15, pages 1043-44 (1948)). It deals with bounds for the characteristic roots of matrices with positive (nonnegative) elements, and with bounds for multiple roots.
\end{abstract}

This note is a continuation of an earlier one [1]. ${ }^{1}$ There the position of the characteristic roots of an $n \times n$ matrix $A=\left(a_{i k}\right)$ inside or on the boundary of the $n$ circles $C_{i}$ with centers $a_{i i}$ and radii $A_{i}=\sum_{k \neq i}\left|a_{i k}\right|$
was studied.

In particular, it was shown in [1] that for $n=2$ the common part of the circles cannot contain a root unless it is a common boundary point. This fact is not true for $n>2$ as is, for example, shown by the matrix

$$
\left(\begin{array}{rrr}
0 & 1 & 0 \\
0 & 0 & 1 \\
0 & -15 & -8
\end{array}\right) \text {, }
$$

which has the roots $0,-3,-5$. The root 0 is contained in all three circles. It can however be shown that an analogue of the situation for $n=2$ holds if further conditions are imposed on the elements $a_{i k}$.

Theorem 1. The dominant root of a matrix of positive elements cannot be a common point of all $n$ circles $C_{i}$ unless it is a common boundary point of at least two of the circles.

Proof. It is known [2] that the dominant root $\lambda$ of such a matrix is real and positive and that the corresponding characteristic vector $x_{1}, \cdots, x_{n}$ can be chosen in such a way that all its components are positive. Consider then the equation

$$
a_{i 1} x_{1}+\cdots+\left(a_{i i}-\lambda\right) x_{i}+\cdots+a_{i n} x_{n}=0,
$$

or

$$
\left(\lambda-a_{i i}\right) x_{i}=\sum_{k \neq i} a_{i k} x_{k} .
$$

As the right-hand side of (2) is positive, it follows that

$$
\lambda-a_{i i}>0 .
$$

Equation (2) implies that

$$
\left(\lambda-a_{i i}\right) x_{i} \geq A_{i} \min _{k \neq i} x_{k} .
$$

Let $\lambda$ be an inner point of all circles $C_{i}$, that is,

$$
\lambda-a_{i i}<A_{i}, \quad i=1, \cdots, n .
$$

It follows from (4) that

$$
x_{i}>\min _{k \neq i} x_{k}, \quad i=1, \cdots, n .
$$

\footnotetext{
${ }_{1}$ Figures in brackets indicate the literature references at the end of this paper.
}

This is not possible. Hence $\lambda$ cannot be an inner point of all $C_{i}$. Assume then that $\lambda$ was on the boundary of at least one circle $C_{i}$, say $C_{1}$, and an inner or boundary point of all other circles. Then relation (4) for $i=1$ implies that

Let

$$
x_{1} \geq \min _{k \neq 1} x_{k}
$$

$$
\min _{k \neq 1} x_{k}=x_{2},(\text { say })
$$

and consider relation (4) for $i=2$. This implies that either

$$
\lambda-a_{22}=A_{2} \text { and } x_{2} \geq \min _{k \neq 2} x_{k}
$$

or

$$
\lambda-a_{22}<A_{2} \text { and } x_{2}>\min _{k \neq 2} x_{k} .
$$

However, relations (6), (7), (9) lead to a contradiction and so (8) holds which means that $\lambda$ is on the boundary of $C_{2}$.

It can be shown that the dominant root of a matrix of positive elements can lie inside of $n-1$ circles and outside of one circle without touching the boundaries. The following example of this was communicated to the author by A. Ostrowski:

Define $a_{i k}(i \neq k)$ as arbitrary positive numbers. Take $\lambda$ sufficiently large and positive and define $a_{i i}$ by

$$
\begin{aligned}
& a_{i i}=\lambda-\frac{1}{2} a_{i 1}-\sum_{\substack{k=2 \\
k \neq i}}^{n} a_{i k}>0, i>1, \\
& a_{11}=\lambda-2 \sum_{k=2}^{n} a_{1 k}>0 .
\end{aligned}
$$

It follows that $\lambda$ is a characteristic root of the matrix $\left(a_{i k}\right)$ and that the corresponding vector is $\left(\frac{1}{2}, 1, \cdots, 1\right)$. Hence $\lambda$ is the dominant root, as the other roots cannot have vectors with positive coordinates only [2].

Two generalizations of theorem 1 are possible, one to matrices with nonnegative elements instead of positive ones. These matrices have as one of their dominant roots a nonnegative real number, and the corresponding vector can be chosen to have nonnegative components. Hence, all the above arguments can be repeated, as long as equations (2) do not involve any equation of the form $0=0$. This 
could only occur, if the matrix $A$ can be transformed to the form

$$
\left(\begin{array}{ll}
P & O \\
U & Q
\end{array}\right)
$$

by the same permutation of the rows and columns [1] and where $P$ and $Q$ are square matrices, and 0 consists of zeros. Thus the following theorem holds:

Theorem 2. Let $A$ be a matrix of nonnegative elements which cannot be transformed to the form

$$
\left(\begin{array}{ll}
P & O \\
U & Q
\end{array}\right)
$$

by the same permutation of the rows and columns. Then the real dominant root of $A$ cannot be a common point of all $n$ circles $C_{i}$ unless it is a common boundary point of at least two of these circles.

Consider now matrices whose main diagonal elements are arbitrary real, but whose off-diagonal, elements are positive (nonnegative). It is known $[3,4]$ that such a matrix has as root with largest real part a real positive (nonnegative) number and the components of the corresponding vectors can be chosen to be all positive (nonnegative). It is clear that theorems 1 and 2 can be generalized to these matrices.

The remaining two theorems concern multiple roots of general matrices. It is easy to find examples of matrices with a multiple root that is contained as inner or boundary point in only one circle $C_{i}$. It can, however, be proved that in this case the rank of the matrix

$$
\left(a_{i k}-\lambda \delta_{i k}\right) \text { is } n-1 .
$$

Theorem 3. A characteristic root $\lambda$ which is an inner or boundary point of only one circle $C_{i}$ cannot have two independent characteristic vectors corresponding to it.

Proof. Assume that $\left|a_{11}-\lambda\right| \leq A_{1}$ and $\left|a_{i i}-\lambda\right|>A_{i}$ for $i \neq 1$. Consider any characteristic vector $x_{1}, \ldots$, $x_{n}$ which corresponds to $\lambda$. Then the relations

$$
\left|a_{i i}-\lambda\right|\left|x_{i}\right| \leq A_{i} \max _{k \neq i}\left|x_{k}\right|
$$

hold. For $i>1$ they imply that

$$
\left|x_{i}\right|<\max _{k \neq i}\left|x_{k}\right| \cdot
$$

Hence

$$
\left|x_{1}\right|=\max _{k}\left|x_{k}\right|>\left|x_{i}\right| \text { for } i>1 .
$$

This eliminates the possibility of a vector $y_{1}, \ldots, y_{n}$ that corresponds to $\lambda$ and is independent of $x_{1}, \ldots$, $x_{n}$, because otherwise a linear combination of both vectors could be found in which the first component is 0 and which does not vanish identically.
Theorem 4. If $A$ has a characteristic root $\lambda$ of multiplicity $n-1$ and with $n-1$ independent characteristic vectors then $\lambda$ is contained in at least $n-1$ circles $C_{i}$.

Proof. The matrix $\left(a_{i k}-\lambda \delta_{i k}\right)$ has the same vectors as $\left(a_{i k}\right)$ and as roots the numbers $\mu-\lambda$ when $\mu$ runs through all roots of $\left(a_{i k}\right)$. The circles that correspond to the matrix $\left(a_{i k}-\lambda \delta_{i k}\right)$ have the same radii as the original ones, but their centers are moved from $a_{i i}$ to $a_{i i}-\lambda$. Hence, we may restrict ourselves to the case where $\lambda=0$. In this case the matrix can be transformed to the form

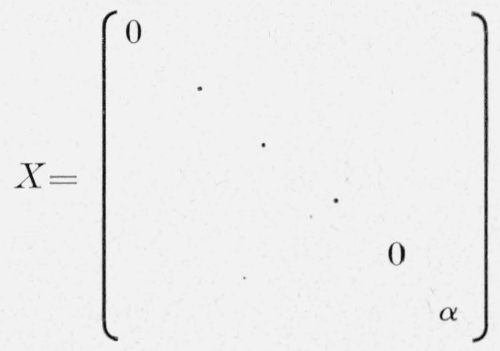

by means of a nonsingular matrix $U=\left(u_{i k}\right)$. Denote by $\Delta$ the determinant $\left|u_{i k}\right|$ and by $U_{i k}$ the cofactor of $u_{i k}$. It is then easy to see that the original matrix $\left(a_{i k}\right)=U X U^{-1}$ is of the form

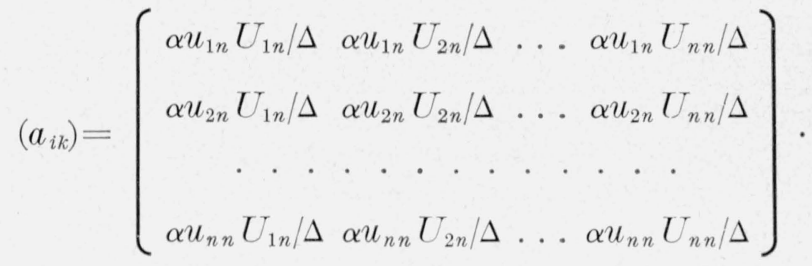

Assume now that 0 lies outside one of the circles, say $C_{1}$. This implies

$$
\left|U_{1 n}\right|>\sum_{k \neq i}\left|U_{k n}\right| \cdot
$$

This, however, implies that

$$
\left|U_{i n}\right|<\sum_{k \neq i}\left|U_{k n}\right|, \quad i=2, \ldots, n .
$$

From this it follows that 0 lies inside all circles $C_{i}$, $i=2, \ldots, n$, which proves the theorem.

[1] O. Taussky, Bounds for characteristic roots of matrices, Duke Math. J. 15, 1043 (1948).

[2] G. Frobenius, Ưber Matrizen aus positiven elementen, Berlin Sitzb. p. 471 (1908) and 514 (1909); Über Matrizen aus nicht negativen elementen, Berlin Sitzb. p. $456(1912)$.

[3] H. Rohrbach, Bemerkungen zu einem determinantensatz, Jahresber. Deut. Math. Ver. 40, 49 (1931).

[4] Z. Szmydtówna, Sur les racines caractéristiques et sur les directions caractéristiques de certains matrices, Ann. Soc. Pol. Math. 22, 235 (1949).

Washington, September 20, 1950. 